$\mathrm{PM} / 93-37$

THES-TP 93/11

October 1993

\title{
Unitarity Constraints for Transverse Gauge Bosons at LEP and Supercolliders $\dagger$
}

\author{
G.J. Gounaris \\ Department of Theoretical Physics \\ University of Thessaloniki, Greece \\ J. Layssac, F.M. Renard \\ Physique Mathématique et Théorique, \\ CNRS-URA 768, Université Montpellier II \\ F-34095 Montpellier Cedex 5
}

\begin{abstract}
Using asymptotic helicity amplitudes for Vector-Vector, Vector-Higgs and Higgs-Higgs scattering, we establish the unitarity constraints on the $S U(2)_{c}$ conserving and $W_{\mu}$ depending interactions, which at sufficiently high energies may create strong forces among the transverse vector boson and Higgs states. We then derive upper bounds for the couplings of these interactions, which depend on the high energy scale where unitarity is saturated. If e.g. unitarity is saturated at $1 \mathrm{TeV}$, then $\lambda_{W} \lesssim 0.12$ is obtained. The implied relations between the present and future LEP results, and the possible observations of strong effects at supercolliders, are also discussed.
\end{abstract}

$\dagger$ Work supported by the scientific cooperation program between CNRS and EIE. 
At present the main meagre indication for New Physics(NP) beyond the Standard Model(SM) comes from the dark matter problem, the deficit in the solar neutrino flux [1] and some peculiarities in double beta decay spectra [2]. These may be hinting at some non vanishing neutrino masses and/or the existence of majorons or other exotic particles, which clearly belong to NP. On the other hand, for the gauge boson-fermion interactions everything looks quite standard. To some extend, the same is also true for the gauge boson self-interactions, although the present precision results mainly from LEP1, still allow considerable discrepancies from SM. LEP2 and future supercolliders will look at these gauge boson self interactions and scrutinize whether there is any NP hidden there.

There are many ideas concerning the form of the NP. Inevitably, they all involve new degrees of freedom . These degrees of freedom may either be too heavy to be directly produced at the contemplated supercolliders, or they might appear as light weakly interacting particles (like axions, majorons, photinos etc.) that are difficult to be discovered. In both cases it is reasonable to expect that at some level they will affect the gauge boson self interaction, as well as their interaction with the standard physical Higgs particle. It should therefore be fruitfull to look for NP by studying the gauge boson and Higgs sector. In fact this may turn out to be the only way to look for NP in the first of the two cases mentioned above, where no NP particles can be directly produced at the future supercolliders. In such a case the appearance of new strong interactions at sufficiently high energies seems inevitable. And this is the case that we would like to concentrate on in the present work.

Even if all new degrees of freedom are very heavy, there are still many possibilities for the form of the high energy strong interactions induced by NP. In order to reduce them we need a principle. It turns out that such a principle is available. Motivated by the fact that the neutral to charge current ratio satisfies $\rho \simeq 1$, it has been emphasized by many authors that the gauge boson and Higgs self interactions should be invariant under global $S U(2)_{c}$ transformations [3]. Under this assumption, there are two alternative approaches that have been followed.

In the first approach, originally emphasized by Lee Quigg and Thacker [4] and subsequently by others [5, 6], the additional assumption is made that the $S U(2)_{c}$ invariant operators induced by NP depend on the scalar fields only. If the couplings of these operators are sufficiently large, then the NP at the $\mathrm{TeV}$ scale appears in the form of a strong interaction affecting the longitudinal gauge bosons only, while the transverse gauge bosons continue to interact weakly. In this approach, the Higgs particle should be very heavy; i.e. $M_{H} \gtrsim 1 T e V$.

In the second alternative suggested in Refs. [7, 8], no restriction is imposed on the type of the SM fields appearing in the $S U(2)_{c}$ invariant operators induced by NP. Thus the NP operators are allowed to depend on the gauge field $W_{\mu}$ also. Restricting their dimensions to be at most six, there exist only two such operators called $\mathcal{O}_{W}$ and $\mathcal{O}_{U W}$. Provided their couplings are sufficiently large, strong interactions may be generated at the TeV scale, albeit among the transverse gauge bosons now. The mass of the physical

\footnotetext{
${ }^{1}$ The usual Higgs doublet is taken as part of SM and not as part of the new degrees of freedom.
} 
Higgs is not affected by these couplings at tree level. Thus, we may have $M_{H} \sim M_{W}$, so that the longitudinal gauge bosons continue interacting weakly at all energies. On the other hand, if it happens that $M_{H} \gg M_{W}$ and the coupling of either $\mathcal{O}_{W}$ or $\mathcal{O}_{U W}$ is large enough, then the two alternatives coexist; i.e. both the transverse as well as the longitudinal gauge bosons interact strongly at $\mathrm{TeV}$ energies.

The unitarity constraints for the first alternative mentioned above, have been studied long ago [⿴囗十]. Assuming that the Higgs particle is very heavy, these constraints give the energy scale at which the longitudinal gauge boson amplitudes reach unitarity values. Many authors have studied the signatures of these strong interactions at various supercollider experiments [5, 6, 9].

In the present paper we study the unitarity constraints in the second alternative approach, where the high energy strong interactions affect the transverse gauge bosons only. To this aim we study in detail the $\mathcal{O}_{W}$ and $\mathcal{O}_{U W}$ contributions to the vector boson and Higgs scattering amplitudes. To isolate the second alternative from the first, we assume $M_{H} \sim M_{W}$. As in [4, 5] we work with tree-level amplitudes. We then find that if unitarity is saturated at the $\mathrm{TeV}$ scale or higher, then the couplings of the operators $\mathcal{O}_{W}$ and $\mathcal{O}_{U W}$ are very efficiently constrained. This constraint is in fact so strong, that if $\mathcal{O}_{W}$ is indeed observed at LEP2, then it should be concluded that either strong interactions must appear at about $1 \mathrm{TeV}$, or new particles below $1 \mathrm{TeV}$.

More spectacularly, our unitarity constraints appear to be much more efficient than those obtained from radiative corrections measurable at LEP1 [10]. Of similar nature are also the unitarity constraints that we find for $\mathcal{O}_{U W}$. We should also mention that these constraints are more stringent than those obtained in [11] by studying fermion-fermion annihilation to $W^{+} W^{-}, W Z$ and $W \gamma$, even though no $S U(2) \times U(1)$ gauge invariance was imposed there, which should certainly further weaken them. Below we derive our results and subsequently discuss them.

It has been shown in 8 that, if we impose $\mathrm{CP}$ and $S U(2)_{c}$ symmetries as well as $S U(2) \times U(1)$ gauge invariance, and we restrict to operators of dimension up to six, then the effective lagrangian describing boson production at the various colliders is completely described by

$$
\begin{gathered}
\mathcal{L}=\mathcal{L}_{S M}+\mathcal{L}_{N P} \\
\mathcal{L}_{S M}=-\frac{1}{2}\left\langle W_{\mu \nu} W^{\mu \nu}\right\rangle-\frac{1}{4} B_{\mu \nu} B^{\mu \nu}+\frac{v^{2}}{4}\left\langle D_{\mu} U D^{\mu} U^{\dagger}\right\rangle \\
-\frac{v^{2} M_{H}^{2}}{8}\left(\frac{1}{2}\left\langle U U^{\dagger}\right\rangle-1\right)^{2}+\text { fermionic terms } \\
\mathcal{L}_{N P}=\lambda_{W} \frac{g_{2}}{M_{W}^{2}} \mathcal{O}_{W}+d \mathcal{O}_{U W}
\end{gathered}
$$


where

$$
\begin{gathered}
\mathcal{O}_{W}=\frac{1}{3 !}\left(\vec{W}_{\mu}{ }^{\nu} \times \vec{W}_{\nu}{ }^{\lambda}\right) \cdot \vec{W}_{\lambda}{ }^{\mu}=-\frac{2 i}{3}\left\langle W^{\nu \lambda} W_{\lambda \mu} W_{\nu}^{\mu}\right\rangle \\
\mathcal{O}_{U W}=\left\langle\left(U U^{\dagger}-1\right) W^{\mu \nu} W_{\mu \nu}\right\rangle, \\
U=(\widetilde{\Phi}, \Phi) \frac{\sqrt{2}}{v} .
\end{gathered}
$$

Here $\Phi$ is the standard Higgs doublet, and the definitions $v^{-2}=\sqrt{2} G_{F}, \widetilde{\Phi}=i \tau_{2} \Phi^{*}$ and $\langle A\rangle \equiv \operatorname{Tr} A$ are used.

Since the operators $\mathcal{O}_{W}$ and $\mathcal{O}_{U W}$ have a high dimension $(\operatorname{dim}=6)$, they tend to create strong forces at high energies. It turns out that these forces affect only the transverse gauge bosons. In order to separate them from the extensively studied strong forces among the longitudinal gauge bosons, which are generated by the scalar sector in (2) in case the Higgs particle is heavy [3, 5, 6], we concentrate here on the situation of a "light" Higgs; i.e. $M_{H} \sim M_{W}$. In this scenario, only the transverse gauge bosons may interact strongly at the few $\mathrm{TeV}$ scale.

The unitarity constraints for $\mathcal{O}_{W}$ and $\mathcal{O}_{U W}$ come from studying four body amplitudes involving gauge bosons and the physical Higgs as external particles. Convenient approximate expressions, valid at the per cent level for CM energies $\sqrt{s} \gtrsim 1 \mathrm{TeV}$, have been recently found for the gauge boson amplitudes $V V \rightarrow V V$, with $V=W^{+}, W^{-}, Z$ or $\gamma$ [12], and the amplitudes for $V V \rightarrow V H$, and $V V \rightarrow H H$ involving external Higgs particles [13. Using this explicit form of the covariant helicity amplitudes at high energy, together with the well known partial wave expansion [14],

$$
F\left(\lambda_{1} \lambda_{2} \rightarrow \lambda_{3} \lambda_{4}\right)=16 \pi \sum_{j}\left(j+\frac{1}{2}\right) D_{\lambda_{1}-\lambda_{2}, \lambda_{3}-\lambda_{4}}^{j *}(\phi, \theta, 0)\left\langle\lambda_{3} \lambda_{4}\left|T^{j}\right| \lambda_{1} \lambda_{2}\right\rangle
$$

we obtain the partial wave amplitudes $T^{j}$. Note that in the r.h.s. of (7) a factor of $\sqrt{2}$ should be inserted, for any initial or final state channel involving identical particles. For our unitarity study, it is sufficient to calculate these amplitudes for the lowest total angular momentum $j$ only, since these should be the most sensitive ones to possible strong interactions. In (7) $\lambda_{i}$ denote the helicities, and the normalization of the partial wave amplitude $T^{j}$ is the same as in $2 e^{i \delta} \sin \delta$, so that the unitarity limit for elastic processes is given by $\left|T^{j}\right| \leq 2$. A stronger constraint could be derived by imposing $\left|\operatorname{Re}\left(T^{j}\right)\right| \leq 1$, which could be justified if one would know the analyticity structure of the true amplitudes at high energy. In order to be conservative, we shall not use this more stringent constraint $\mathrm{I}^{2}$. In the following we shall also treat each effective operator separately and ignore possible, model dependent, cancellation effects between $\mathcal{O}_{W}$ and $\mathcal{O}_{U W}$.

We first study the unitarity constraints from the process $W^{+} W^{+} \rightarrow W^{+} W^{+}$, characterized by total charge $Q=2$ in the initial or final state. For $j=0$, only channels where

\footnotetext{
${ }^{2}$ Had we used it, then the r.h.s. of the inequalities (11-16) below would be smaller by a factor $\sqrt{2}$.
} 
both $W^{+}$'s have the same helicity, feel strongly the $\mathcal{O}_{W}$ and $\mathcal{O}_{U W}$ interactions. Identifying

$$
\left|W^{+} W^{+}++\right\rangle \rightarrow\left(\begin{array}{l}
1 \\
0
\end{array}\right), \quad\left|W^{+} W^{+}--\right\rangle \rightarrow\left(\begin{array}{l}
0 \\
1
\end{array}\right),
$$

we find [12] that the $\mathcal{O}_{W}$ contribution at $\sqrt{s} \gtrsim 1 T e V$ is approximately given by

$$
T^{j=0}=-\frac{\alpha}{24 s_{W}^{2}}\left(\frac{\lambda_{W} s}{M_{W}^{2}}\right)^{2}\left(\begin{array}{cc}
0 & 1 \\
1 & 0
\end{array}\right),
$$

while the $\mathcal{O}_{U W}$ contribution is [12, 13]

$$
T^{j=0}=\frac{\alpha d^{2} s}{4 M_{W}^{2} s_{W}^{2}}\left(\begin{array}{ll}
0 & 1 \\
1 & 0
\end{array}\right) .
$$

Using the eigenvalues of (9) and (10) we then find that the unitarity limits for $\lambda_{W}$ and $d$ are respectively given by

$$
\left|\frac{s \lambda_{W}}{M_{W}^{2}}\right| \lesssim \sqrt{\frac{48 s_{W}^{2}}{\alpha}} \simeq 39
$$

and

$$
\frac{\sqrt{s}}{M_{W}}|d| \lesssim \sqrt{\frac{8 s_{W}^{2}}{\alpha}} \simeq 16
$$

Before discussing the significance of these results for LEP2 and the future supercolliders, we first quote the even stronger unitarity constrains obtained from a similar study of channels with total charge $Q=1$ and $Q=0$ in the incoming and outgoing states. These sectors generally involve a larger number of channels, and thus bigger matrices.

Thus for $\mathcal{O}_{W}$, the dominant $j=0$ partial wave amplitudes in the $Q=1$ sector involve the channels $\left|W^{+} Z++\right\rangle,\left|W^{+} Z--\right\rangle,\left|W^{+} \gamma++\right\rangle,\left|W^{+} \gamma--\right\rangle$ [12]. The $T^{0}$ matrix is $4 \times 4$, and its largest eigenvalue gives

$$
\left|\frac{s \lambda_{W}}{M_{W}^{2}}\right| \lesssim \sqrt{\frac{24 s_{W}^{2}}{\alpha}} \simeq 27.5 \quad .
$$

Similarly for the $Q=0$ sector, the relevant channels are $\left|W^{+} W^{+}++\right\rangle,\left|W^{+} W^{+}--\right\rangle$, $|Z Z++\rangle,|Z Z--\rangle,|Z \gamma++\rangle,|Z \gamma--\rangle,|\gamma \gamma++\rangle,|\gamma \gamma--\rangle$ [12], implying an 8x8 T-matrix whose largest eigenavalue gives

$$
\left|\frac{s \lambda_{W}}{M_{W}^{2}}\right| \lesssim \sqrt{\frac{12 s_{W}^{2}}{\alpha}} \simeq 19
$$


Finaly from the $j=1$ amplitude we get

$$
\left|\frac{s \lambda_{W}}{M_{W}^{2}}\right| \lesssim \sqrt{\frac{96 s_{W}^{2}}{5 \alpha}} \simeq 25
$$

for both the $Q=1$ and $Q=0$ cases. We note for $Q=1$ the contributing channels are again $\left|W^{+} Z++\right\rangle,\left|W^{+} Z--\right\rangle,\left|W^{+} \gamma++\right\rangle,\left|W^{+} \gamma--\right\rangle$; while in the $Q=0$ case only the two channels $\left|W^{+} W^{-}++\right\rangle$and $\left|W^{+} W^{-}-{ }^{-}\right\rangle$are involved [12].

We now turn to $\mathcal{O}_{U W}$. For $j=0$ and $Q=1$, the dominant contributions arise only from the same channels as in the corresponding $\mathcal{O}_{W}$ case [12, 13], and the largest eigenvalue of the relevant $T^{0}$ matrix gives again the same result as in (12). On the other hand for $Q=0$, we need to calculate an $11 \times 11 T^{0}$ matrix involving the same 8 channels as in the corresponding $\mathcal{O}_{W}$ case, and in addition the channels $\left|W^{+} W^{-} L L\right\rangle,|Z Z L L\rangle$ (containing longitudinal gauge bosons) and $|H H\rangle$. Using the amplitudes given in [12, 13] we get

$$
|d| \lesssim 17.6 \frac{M_{W}^{2}}{s}+2.43 \frac{M_{W}}{\sqrt{s}}
$$

The most stringent constraints coming from eq.(14) and (16) are plotted in Fig.1.

The unitarity constraints given in (11-16) involve products of anomalous couplings times the energy of the processes, in linear or quadratic forms. The (approximate) equality signs in (11-16) determine the CM energy $\sqrt{s}$ where unitarity is first saturated. They indicate the intuitively obvious result that as the the couplings $\lambda_{W}$ or $d$ (of the high dimension interactions) become smaller, higher energies are needed in order to reach the unitarity bound. We also note that in deriving the $\mathcal{O}_{W}$ constraints it was sufficient to retain only terms proportional to $\left(\lambda_{W} s / M_{W}^{2}\right)^{2}$ in the amplitudes, while in the $\mathcal{O}_{U W}$ case both the linear and quadratic $d$ contributions were important.

Since the constraints (11-16) are based on the separate treatment of the operators $\mathcal{O}_{W}$ and $\mathcal{O}_{U W}$, they establish the conditions under which each of these operators may be treated perturbatively. If we had treated instead only appropriate combinations of $\mathcal{O}_{W}$ and $\mathcal{O}_{U W}$, then the $\lambda_{W}-d$ domains allowed by the unitarity of the boson-boson amplitudes would be enlarged, but only due to strong cancellations between two large non-perturabative contributions to these amplitudes. The perturbative treatment of $\mathcal{O}_{W}$ and $\mathcal{O}_{U W}$ separately, would not be guaranteed in that case. Thus, such results could not be used to discuss the reliability of the perturbative treatment of quantities like e.g. the radiative corrections to the LEP1 measurements (see below), where only one of the above operators can contribute.

Let us now discuss the interpretation of these results.

The first remark consists in assuming that the values of the couplings $\lambda_{W}$ and $d$ are fixed by some model. For example one can just assume that they are determined by a characteristic scale $\Lambda_{s}$ originating from the dynamics of a strongly interacting system, so 
that we may expect

$$
\frac{\lambda_{W}}{M_{W}^{2}} \sim \frac{\sqrt{4 \pi}}{\Lambda_{s}^{2}} \quad, \quad \frac{d}{M_{W}} \sim \frac{\sqrt{4 \pi}}{\Lambda_{s}}
$$

where $\Lambda_{s} \gtrsim 1 T e V$. Combining (17) with (11-16) we get the allowed range for the CM energies of the various scattering processes, so that they lie below the point where unitarity effects show up. This saturation energy is obviously of the order of $\Lambda_{s}$. Thus, from e.g. our most stringent bound for $\mathcal{O}_{W}$ given in (14) we get

$$
s \lesssim \sqrt{\frac{12 s_{W}^{2}}{4 \pi \alpha}} \Lambda_{s}^{2} \simeq 5.4 \Lambda_{s}^{2} .
$$

The second remark concerns the lowest order contributions of $\mathcal{O}_{W}$ or $\mathcal{O}_{U W}$, to logarithmically divergent one-loop radiative corrections. In such a case the role of $s$ in (11-16) is played by the radiative cut-off $\Lambda^{2}$. Thus, using e.g. (14), we conclude that in order the $\mathcal{O}_{W}$ one-loop radiative correction to be reliable, the coupling $\lambda_{W}$ and the cut-off $\Lambda$ should satisfy the constrain

$$
\left|\frac{\Lambda^{2} \lambda_{W}}{M_{W}^{2}}\right| \lesssim \sqrt{\frac{12 s_{W}^{2}}{\alpha}} \simeq 19
$$

which for $\Lambda \lesssim 1 T e V$, implies that

$$
\left|\lambda_{W}\right| \lesssim 0.12
$$

Correspondingly from (16) we would conclude that the one-loop radiative corrections due to $\mathcal{O}_{U W}$ can only be reliable if

$$
|d| \lesssim 17.6 \frac{M_{W}^{2}}{\Lambda^{2}}+2.43 \frac{M_{W}}{\Lambda} \lesssim 0.3
$$

Below we will return to the implications of this result for LEP1 measurements.

Finally, the third remark concerns the direct search for the anomalous couplings $\lambda_{W}$ and $d$ at (low) energy, i.e. at energies much smaller than the characteristic scale where unitarity is saturated. Suppose that such a "low" energy experiment gives a hint for a certain value for $\lambda_{W}$ or $d$. Then the inequalities (11-16) allow us to predict the lowest energy squared $s_{\text {strong }}$ where strong interactions are expected to appear. Thus using (14) for $\lambda_{W}$, we get

$$
s_{\text {strong }} \simeq 19 \frac{M_{W}^{2}}{\left|\lambda_{W}\right|}
$$

Alternatively, the absence of a direct effect down to the observability limit for $\lambda_{W}$ in a 
low energy experiment, can be transformed into a lower limit for the unset of the new $\mathcal{O}_{W}$ induced strong interaction

$$
s_{\text {strong }} \gtrsim 19 \frac{M_{W}^{2}}{\sup \left|\lambda_{W}\right|},
$$

where sup $\left|\lambda_{W}\right|$ is the upper bound of $\left|\lambda_{W}\right|$. Substituting in (23) the expected or quoted upper bounds for $\left|\lambda_{W}\right|$ that will be observable at the various colliders, we give in Table

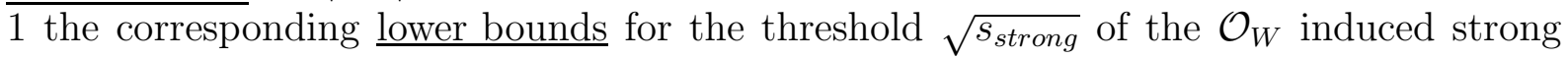
interaction, to which these colliders are sensitive.

\begin{tabular}{|c|c|c|c|}
\hline \multicolumn{4}{|c|}{ Table 1: Observability limits for $\lambda_{w}$ and $\sqrt{s_{\text {strong }}}$} \\
\hline Collider & $\left|\lambda_{W}\right| \lesssim$ & $\sqrt{s_{\text {strong }}}$ & Reference \\
\hline LEP2 $170 \mathrm{GeV}$ & 0.14 & $0.9 \mathrm{TeV}$ & 15 \\
LEP2 $230 \mathrm{GeV}$ & 0.06 & $1.4 \mathrm{TeV}$ & 15 \\
NLC $0.5 \mathrm{TeV}$ & 0.008 & $4 \mathrm{TeV}$ & 16 \\
NLC $1 \mathrm{TeV}$ & 0.002 & $8 \mathrm{TeV}$ & 16 \\
SSC/LHC & 0.01 & $3.5 \mathrm{TeV}$ & 8 \\
\hline
\end{tabular}

Corresponding constraints for $\mathcal{O}_{U W}$ are derived using (16) together with the result that $\mathcal{O}_{U W}$ should be observable at SSC/LHC provided $|d| \gtrsim 0.1$ [12]. From this we conclude that $\mathrm{SSC} / \mathrm{LHC}$ is sensitive to the $\mathcal{O}_{U W}$ induced strong interaction, provided its threshold is at $\sqrt{s_{\text {strong }}} \lesssim 2.5 \mathrm{TeV}$. These results are further illustrated in Fig.1.

Precision tests at LEP1 were also used to set bounds on the anomalous coupling $\lambda_{W}$ of $\mathcal{O}_{W}$ [10, 17]. At the $\mathrm{Z}$ peak, $\mathcal{O}_{W}$ contributes only through 1-loop radiative corrections to the vector boson self-energies and the vector-fermion-fermion vertices. The use of effective operators within loops requires some care as soon as the high energy part of the loop integral is not negligible, and especially when it is divergent. This was extensively discussed in [10, 17]. The contribution of $\mathcal{O}_{W}$ to $\mathrm{Z}$ peak observables turns out to be logarithmically divergent; i.e. it varies like $\lambda_{W} \log \left(\frac{\Lambda}{M_{W}}\right)$, where $\Lambda$ is the cut-off. Thus the bound obtained from $\mathrm{Z}$ peak measurements of $\lambda_{W}$ corresponds to ignoring all contributions to the loop integral, which are associated with energies higher than $\Lambda$. So a first remark is that one can appreciate the sensitivity to this unknown sector by varying $\Lambda$. A second remark stems from the observation that the LEP1 result for $\Lambda=1 \mathrm{TeV}$ and $M_{H}=100 \mathrm{GeV}$ 10, 17]

$$
\left|\lambda_{W}\right| \lesssim 0.6 \quad
$$

casts doubts on the reliability of the one-loop treatment of $\mathcal{O}_{W}$, to the extend that it allows a violation of $(19,20)$.

The fact that the anomalous $\mathcal{O}_{W}$ and $\mathcal{O}_{U W}$ operators, even with very small couplings, lead at high energy to strong interactions among transverse W states, should always be kept in mind. It is just a consequence of the high dimensions of these operators. In the present work we have gone beyond this though, by giving the relations connecting the 
low energy relics (i.e. the anomalous $\lambda_{W}$ and d couplings) to the direct manifestations of unitarity saturation at high energies.

Such a situation may be compared to the one mentioned in the introduction, - the first approach to $S U(2)_{c}$ realization-, where strong $W_{L}$ interactions exist but are not seen at low energy. Several studies of the effect of the anomalous couplings $\left(\kappa_{Z}, \kappa_{\gamma}, \delta_{Z}, \ldots\right)$ on the $W_{L}$ properties have been done in this framework, using the chiral structure of the scalar sector [5, 9]. It is implicitly assumed in this framework, that the anomalous couplings have their origin in the dynamics associated with the mass generation of the $\mathrm{W}$ bosons.

In our case the situation may be different. Although the $\mathrm{W}$ mass generation may still be the motivation for modifying the SM, the New Physics may also affect directly the $W_{T}$ interactions. Such a phenomenon has also appeared previously as a consequence of extending the gauge sector. In our case it is generated by $S U(2)_{c}$ invariance, which had not been imposed so strictly before. In this paper we established some milestones for the resulting properties. We have shown that a new type of a strongly interacting sector affecting transverse $\mathrm{W}$ states may be generated. The magnitude of the anomalous $W_{T}$ couplings observed in a "low" energy experiment, is then directly related to the high energy scale at which strong interactions should appear. Moreover, we have found that even an upper bound on such an anomalous coupling at low energies, implies a useful lower bound for the scale of the expected unitarity effects. These relations turn out to produce remarkable connections between LEP2 measurements and supercollider experiments.

\section{Acknowledgements}

One of us (GJG) wishes to thank the Laboratoire de Physique Mathématique et Théorique de l'Université Montpellier II, for the hospitality and kind help he received during his stay in Montpellier. 


\section{References}

[1] R.Davis, D.S.Harmer and K.C.Hoffman, Phys. Rev. Lett. 20 (1968) 1205 ; R.Davis in Proc. 21st Intern. Cosic Ray Conf., ed. R.J.Protheroe, Vol 12 (Univ. Adelaide, Australia, 1990). GALLEX Colab. Phys. Lett. B285 (1992) 376 . K.S.Hirata et.al. Phys. Rev. D44 (1991) 2241 . SAGE Colab. Phys. Rev. Lett. 67 (1991) 3332.

[2] M.Moe et.al.Nucl. Phys. (Proc. Suppl.) B31 (1993); F.T. Avignone et.al. Phys. Lett. B256 (1991) 559 .

[3] T.Appelquist in "Gauge Theories and Experiments at High Energies", ed. by K.C. Brower and D.G. Sutherland, Scottish University Summer School in Physics, St. Andrews (1980).

[4] B.W.Lee, C.Quigg and H.Thacker, Phys. Rev. D16 (1977) 1519 .

[5] M.S.Chanowitz and M.K.Gaillard, Nucl. Phys. B261 (1985) 379 ; M.S.Chanowitz, Ann.Rev.Nucl.Part.Sci.38(1988)323.

[6] Ken-ichi Hikasa, Inv. talk at Workshop on Physics with Linear Colliders, Finland (1991). See also A.Dobado, M.J.Herrero and J.Terron, Z. Phys. C50 (1991) 205 , Z. Phys. C50 (1991) 465 and refences therein.

[7] G.J.Gounaris and F.M.Renard, Z. Phys. C59 (1993) 133 .

[8] G.J.Gounaris and F.M.Renard, Z. Phys. C59 (1993) 143 .

[9] J.Bagger, S.Dawson and G.Valencia, Nucl. Phys. B399 (1993) 364 .

A.Falk, M.Luke and E.Simmons, Nucl. Phys. B365 (1991) 523 .

[10] A.De Rujula et al, Nucl. Phys. B384 (1992) 3 .

[11] U.Baur and D. Zeppenfeld, Phys. Lett. B201 (1988) 383 .

[12] G.J.Gounaris, J.Layssac and F.M.Renard, preprint PM/93-26, THES-TP 93/8, to be published.

[13] G.J.Gounaris J. Layssac and F.M.Renard, in preparation.

[14] M.Jacob and G.C.Wick, Ann.Phys. 7 (1959) 404.

[15] M.Bilenky, J.L.Kneur, F.M. Renard and D. Schilknecht, Nucl. Phys. B409 (1993) 22 .

[16] G.J.Gounaris et al, in Proc. of the Workshop on $e^{+} e^{-}$Collisions a $500 \mathrm{GeV}$ : The Physics Potential, DESY 92-123B(1992), p.735, ed. P.Zerwas. M.Bilenky et al, BITP in preparation. 
[17] K.Hagiwara et al, Phys. Lett. B283 (1992) 353 , Phys. Rev. D48 (1993) 2182 . T.Appelquist and G-H.Wu Phys. Rev. D48 (1993) 3235 . 


\section{Figure Captions}

Fig.1 Unitarity constraints for $\lambda_{W}$ and d couplings. The light grey $\left(\lambda_{W}\right)$ and dark grey $(d)$ domains show the regions where strong interaction effects should be prominent. We have indicated the indirect LEP1 bound on $\lambda_{W}$ and the observability limits for direct observation expected at various machines. 